\title{
Estimating ambiguity fixed satellite orbit, integer clock and daily bias products for GPS L1/L2, L1/L5 and Galileo E1/E5a, E1/E5b signals
}

\author{
Bingbing Duan ${ }^{1}$ (1) $\cdot$ Urs Hugentobler $^{1} \cdot$ Inga Selmke $^{1} \cdot$ Ningbo Wang $^{1}$
}

Received: 19 November 2019 / Accepted: 11 March 2021 / Published online: 31 March 2021

(c) The Author(s) 2021

\begin{abstract}
Ambiguity resolution of a single receiver is becoming more and more popular for precise GNSS (Global Navigation Satellite System) applications. To serve such an approach, dedicated satellite orbit, clock and bias products are needed. However, we need to be sure whether products based on specific frequencies and signals can be used when processing measurements of other frequencies and signals. For instance, for Galileo E5a frequency, some receivers track only the pilot signal (C5Q) while some track only the pilot-data signal (C5X). We cannot compute the differences between C5Q and C5X directly since these two signals are not tracked concurrently by any common receiver. As code measurements contribute equally as phase in the Melbourne-Wuebbena (MelWub) linear combination it is important to investigate whether C5Q and C5X can be mixed in a network to compute a common satellite MelWub bias product. By forming two network clusters tracking Q and X signals, respectively, we confirm that GPS C5Q and C5X signals cannot be mixed together. Because the bias differences between GPS C5Q and C5X can be more than half of one wide-lane cycle. Whereas, mixing of C5Q and C5X signals for Galileo satellites is possible. The RMS of satellite MelWub bias differences between Q and X cluster is about 0.01 wide-lane cycles for both E1/E5a and E1/E5b frequencies. Furthermore, we develop procedures to compute satellite integer clock and narrow-lane bias products using individual dual-frequency types. Same as the finding from previous studies, GPS satellite clock differences between L1/L2 and L1/L5 estimates exist and show a periodical behavior, with a peak-to-peak amplitude of $0.7 \mathrm{~ns}$ after removing the daily mean difference of each satellite. For Galileo satellites, the maximum clock difference between E1/E5a and E1/E5b estimates after removing the mean value is $0.04 \mathrm{~ns}$ and the mean RMS of differences is $0.015 \mathrm{~ns}$. This is at the same level as the noise of the carrier phase measurement in the ionosphere-free linear combination. Finally, we introduce all the estimated GPS and Galileo satellite products into PPP-AR (precise point positioning, ambiguity resolution) and Sentinel-3A satellite orbit determination. Ambiguity fixed solutions show clear improvement over float solutions. The repeatability of five ground-station coordinates show an improvement of more than $30 \%$ in the east direction when using both GPS and Galileo products. The Sentinel-3A satellite tracks only GPS L1/L2 measurements. The standard deviation (STD) of satellite laser ranging (SLR) residuals is reduced by about $10 \%$ when fixing ambiguity parameters to integer values.
\end{abstract}

Keywords Integer satellite clock · Ambiguity resolution · Daily code and phase biases · GPS and Galileo signals · Pilot and data

\section{Introduction}

The IGS (International GNSS Service) has been providing GPS satellite orbit and clock products for more than 20 years

Bingbing Duan

bingbing.duan@tum.de

$\bowtie$ Urs Hugentobler urs.hugentobler@tum.de

1 Institute for Astronomical and Physical Geodesy, Technical University of Munich, Arcisstr 21, 80333 Munich, Germany
(Dow et al. 2009; Johnston et al. 2017). Within the IGS processing, ambiguity parameters are fixed to integer values. The typical approach is to form double-difference between observations that are simultaneously acquired between two satellites and two receivers. Clock errors and biases on both satellite and receiver sides are eliminated, and the doubledifferenced ambiguity parameters can be consequently fixed to integer values (Teunissen et al. 2003). However, satellite clock products need to be estimated by a second run using zero-difference observations (Dach et al. 2009; Prange et al. 2017). In order to make use of the fixed ambiguity parame- 
ters in clock estimation, the GeoForschungsZentrum (GFZ) decomposes each integer double-difference ambiguity into a pseudo zero-difference observation, and then jointly uses them with the real code and phase observations by giving a very tight constraint (Ge et al. 2005; Uhlemann et al. 2015; Deng et al. 2016).

Different than fixing double-difference ambiguities, CNES/CLS (Centre National d'Etudes Spatiales/Collecte Localisation Satellites) estimates dedicated satellite orbit, clock and bias products when fixing zero-difference ambiguities to integer values (Loyer et al. 2012). The advantage is that users can also do ambiguity resolution at zero-difference level by making use of the publicly available CNES/CLS products. However, the clock estimates are slightly inconsistent with respect to the IGS products since they are relative to phase measurements (Montenbruck et al. 2018). To avoid such inconsistencies, GFZ and Wuhan University estimate epoch-wise satellite narrow-lane biases separately and provide such biases together with their satellite products to authorized users (Ge et al. 2008; Geng et al. 2012; Li et al. 2016). Instead of providing float clock and epoch-wise narrow-lane bias products, the Center for Orbit Determination in Europe (CODE) determines daily code and phase biases as observable-specific bias (OSB) terms on each signal and estimates ambiguity fixed clock products (Schaer et al. 2018; Villiger et al. 2019). The advantage is that ambiguity fixed satellite clock products show better consistency to the IGS final products. Furthermore, the correction of OSB products on each signal is more straight forward. In December 2019, CODE made their phase bias products publicly available as routine bias products at https://cddis.nasa.gov/.

From February 2019, the European Galileo constellation has reached a total of 24 satellites, providing positioning, navigation and timing (PNT) services independently. The Galileo signals are transmitted in four frequency bands: E1, E5a, E5b and E6. The current Galileo satellite orbit and clock products in the MGEX (Multi-GNSS Pilot Project) are based on E1 and E5a signals (Montenbruck et al. 2017; Steigenberger and Montenbruck 2017). Since 2018 CODE and CNES/CLS have extended their ambiguity fixed products to Galileo satellites as well (Schaer et al. 2018; Katsigianni et al. 2019a, b). The performance combining GPS and Galileo satellites together in the PPP-AR applications are analyzed by Paziewski and Wielgosz (2015), Li et al. (2018), Xiao et al. (2019). In fact, for the frequency L5, E5a and E5b, some receivers track only pilot signal Q while some track only pilot-data signal $\mathrm{X}$. These two signals are not tracked concurrently by any common receiver. Therefore, the differences of code and phase biases between Q and X need to be considered in the satellite bias estimation.

The first new GPS III satellite was launched on 23 December 2018. The space vehicle number (SVN) and pseudo random noise (PRN) number are identified as SVN 74 and
PRN 04, respectively. Same as GPS Block IIF and Galileo satellites, GPS III satellites provide signals on more than two frequencies. With such an advantage, we may develop a new approach for ambiguity resolution by making use of triplefrequency (Geng and Bock 2013; Li et al. 2019). However, satellite clock estimates are based on one type dual-frequency ionosphere-free linear combination. We need to make sure whether the employed clock products are suitable for the other dual-frequency observations that are not the same as in the clock estimation. As demonstrated by Montenbruck et al. (2012), an apparent inconsistency of the L1, L2 and L5 carrier phase measurements at the $10 \mathrm{~cm}$ level was identified for one GPS Block IIF satellite due to the thermally dependent inter-frequency bias. Therefore, inter-frequency clock bias (IFCB) need to be considered when applying the GPS L1/L2 satellite clock products into L1/L5 observations (Pan et al. 2017). For the first GPS III satellite, Thoelert et al. (2019) show that such thermally induced variations are no longer observed, but the IFCB values still cannot be ignored. Also, as introduced by the Galileo Interface Control Document (ICD), each Galileo satellite broadcasts both E1/E5a and E1/E5b dual frequency clock corrections.

Based on these, the main goal of this contribution is as follows. First, analyze the differences between $\mathrm{Q}$ and $\mathrm{X}$ signals for both GPS and Galileo satellites. Then, estimate ambiguity fixed satellite orbit, clock and daily bias products using GPS L1/L2 and L1/L5, Galileo E1/E5a and E1/E5b signals, respectively. Finally, introduce all the estimated products into ground station positioning and Sentinel-3A satellite orbit determination.

\section{Ambiguity resolution with zero-difference observations}

Pseudorange $(P)$ and phase $(L)$ observations between a receiver (subscript $r$ ) and a satellite (superscript $s$ ) on one frequency $l$ are described as

$$
\begin{aligned}
P_{r, l}^{s}= & \rho_{r, l}^{s}+c\left(d t_{r}-d t^{s}\right)+c\left(d_{r, l}-d_{l}^{s}\right)+I_{r, l}^{s}+T_{r}^{s}+e_{r, l}^{s} \\
L_{r, l}^{s}= & \rho_{r, l}^{s}+c\left(d t_{r}-d t^{s}\right)+c\left(b_{r, l}-b_{l}^{s}\right)-I_{r, l}^{s}+T_{r}^{s}+ \\
& \lambda_{l} N_{r, l}^{s}+\varepsilon_{r, l}^{s}
\end{aligned}
$$

where $\rho$ denotes the geometric distance, $c$ the speed of light, $d t_{r}$ and $d t^{s}$ the receiver and satellite clock offsets, $I$ the ionospheric delay, $T$ the tropospheric delay, $\lambda$ the wavelength, $N$ the phase ambiguity, $e_{r, l}^{s}$ and $\varepsilon_{r, l}^{s}$ the other error terms of pseudorange and phase observations, for instance the relativistic effect and phase wind-up correction. Furthermore, $d$ and $b$ represent the relevant signal-specific biases of pseudorange and phase observations. 
Within zero-difference solutions, ionospheric delays can largely be eliminated by forming the ionosphere-free (IF) linear combination, but at the same time the noise is amplified by about a factor of 3 . When neglecting higher order ionospheric terms, the $I F$ linear combination of the first and second frequency can be expressed as

$$
\begin{aligned}
P_{r, I F}^{s}= & \rho_{r, I F}^{s}+c\left(d t_{r}-d t^{s}\right)+c\left(d_{r, I F}-d_{I F}^{s}\right)+T_{r}^{s}+e_{r, I F}^{s} \\
L_{r, I F}^{s}= & \rho_{r, I F}^{s}+c\left(d t_{r}-d t^{s}\right)+c\left(b_{r, I F}-b_{I F}^{s}\right)+T_{r}^{s} \\
& +I F\left(\lambda_{1} N_{r, 1}^{s}, \lambda_{2} N_{r, 2}^{s}\right)+\varepsilon_{r, I F}^{s}
\end{aligned}
$$

where $I F$ denotes the ionosphere-free linear combination. It may be noted that there is one singularity in the $I F$ phase equation between ambiguity and clock offset parameters for one receiver in one session. Therefore, the datum of clock offsets is determined by pseudorange observations. In the IGS convention, I $F$ code biases of the employed code observations are assumed to be zero while defining the clock datum. For instance, GPS takes P1 (C1W) and P2 (C2W) observations. It may be also noted that the $I F$ ambiguity cannot be expressed in the form $\lambda_{I F} N_{I F}$ where $N_{I F}$ is an integer ambiguity. If we want to resolve such ambiguity parameters we need to first introduce integer wide-lane ambiguities into the $I F$ solution, and then try to fix the resulting so-called narrow-lane ambiguities.

To obtain integer wide-lane ambiguities, the MelbourneWuebbena (MelWub) linear combination is widely used (Melbourne 1985; Wubbena 1985).

$$
\begin{aligned}
& \operatorname{MelWub}\left(P_{r, 1}^{s}, P_{r, 2}^{s}, L_{r, 1}^{s}, L_{r, 2}^{s}\right) \\
& =\frac{f_{1} L_{1}-f_{2} L_{2}}{f_{1}-f_{2}}-\frac{f_{1} P_{1}+f_{2} P_{2}}{f_{1}+f_{2}} \\
& =\lambda_{w l} N_{r, w l}^{s}+\lambda_{w l}\left(b d_{r, w l}-b d_{w l}^{s}\right) \\
& \quad+\varepsilon_{r, w l}^{s}
\end{aligned}
$$

where $N_{r, w l}^{s}$ denotes the wide-lane ambiguity, $b d_{r, w l}$ and $b d_{w l}^{s}$ the corresponding receiver and satellite biases. The MelWub combination is both geometry- and ionospherefree, only ambiguity and bias terms remain in the equation. The wavelength $\lambda_{w l}$ is relatively long (about $86 \mathrm{~cm}$ for GPS $\mathrm{L} 1 / \mathrm{L} 2$ and $75 \mathrm{~cm}$ for Galileo E1/E5a) and it is therefore easy to fix the wide-lane ambiguities to integer values. We need to be aware that code observations contribute equally as phase observations in Eq. (3). $b d_{r, w l}$ and $b d_{w l}^{s}$ consist of both code and phase biases. This bias term is usually described as wide-lane bias, which actually could be confused with the wide-lane bias in the wide-lane phase linear combination. In this contribution, we name this bias term as MelWub bias to make it clear that the bias parameter is determined from the MelWub linear combination and should be used in the MelWub linear combination to resolve wide-lane ambiguities.
The integer wide-lane ambiguities are then introduced into the float IF solution to conduct the narrow-lane ambiguity $N_{r, 1}^{s}$.

$$
\begin{aligned}
L_{r, I F}^{s}= & \rho_{r, I F}^{s}+c\left(d t_{r}-d t^{s}\right)+c\left(b_{r, I F}-b_{I F}^{s}\right)+T_{r}^{s} \\
& +\lambda_{n l}\left(N_{r, 1}^{s}+\frac{\lambda_{w l}}{\lambda_{2}} N_{r, w l}^{s}\right)+\varepsilon_{r, I F}^{s}
\end{aligned}
$$

where $\lambda_{n l}$ denotes the narrow-lane wavelength. Details about wide-lane and narrow-lane ambiguity resolutions are discussed in Sects. 3 and 4.

\section{Satellite MeIWub biases estimation}

Biases in GNSS are caused by hardware delays in satellite and receiver for pseudorange and phase observations. Pseudorange users are affected by the code biases since observations are obtained by measuring the traveling time of the signal between emission and reception. Code biases of all the satellites for one receiver are not the same and cannot be absorbed by the receiver clock parameters. Biases of phase observations can be however absorbed by the ambiguity parameters and will not contaminate the positioning result. Nevertheless, when intending to fix ambiguity parameters to integer values the corresponding phase biases of satellite and receiver must be compensated.

Satellite MelWub biases contain code and phase biases of the same signals used in the MelWub linear combination. For GPS L1/L2 frequency we use $\mathrm{C} 1 \mathrm{~W}, \mathrm{C} 2 \mathrm{~W}, \mathrm{~L} 1 \mathrm{~W}, \mathrm{~L} 2 \mathrm{~W}$ while for GPS L1/L5 frequency we use C1W, C5Q/C5X, L1W, L5Q/L5X signals. For Galileo E1/E5a frequency we use C1C/C1X, C5Q/C5X, L1C/L1X, L5Q/L5X while for Galileo E1/E5b frequency we use C1C/C1X, C7Q/C7X, L1C/L1X, L7Q/L7X signals. As presented by Sleewaegen and Clemente (2018), phase biases between different signals of the same frequency and constellation are nearly the same. It is thus possible to mix different phase signals of the same frequency in the MelWub linear combination. For instance, we assume that phase biases of L5Q and L5X are the same. However, the code bias behavior is quite different. Some receivers track C1C GPS signal instead of C1W and the DCB (differential code bias) values between these two signals can be up to several ns (Montenbruck et al. 2014; Guo et al. 2015; Wang et al. 2016; Håkansson et al. 2017). The C1W-C1C DCB values of GPS satellites can be computed by a station network as C1C and $\mathrm{C} 1 \mathrm{~W}$ can be tracked concurrently by a common receiver. Then, it is possible to correct all the $\mathrm{C} 1 \mathrm{C}$ signals to $\mathrm{C} 1 \mathrm{~W}$ in advance to consistently use the $\mathrm{C} 1 \mathrm{~W}$ signal in the MelWub linear combination. However, for GPS L5, Galileo E5a and E5b frequencies, C5Q and C5X are not tracked by any common receiver. We cannot correct either C5Q to C5X or C5X 


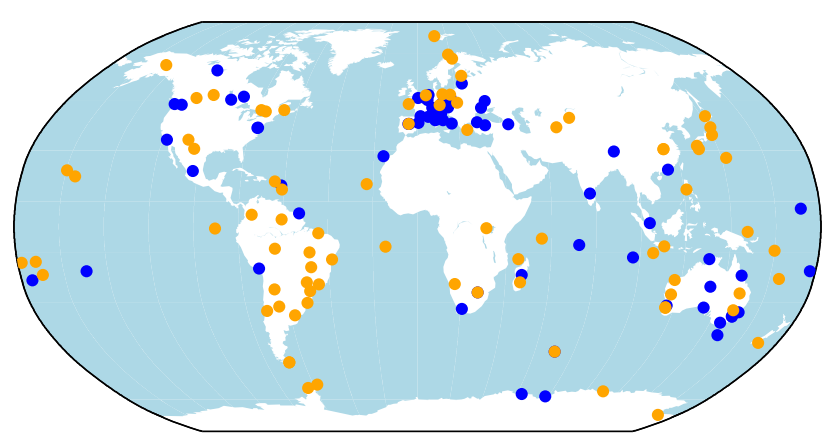

Fig. 1 Ground tracking stations, blue dots denote 65 LEICA and SEPT receivers tracking GPS/Galileo Q signals, yellow dots represent 80 TRIMBLE and JAVAD receivers tracking GPS/Galileo X signals

to $\mathrm{C} 5 \mathrm{Q}$ directly. To investigate whether $\mathrm{Q}$ and $\mathrm{X}$ code signals can be mixed in the MelWub linear combination, we compute the differences of the associated DCB values. For instance, the difference of DCB C1C-C5Q and C1X-C5X can partly reveal the difference between $\mathrm{C} 5 \mathrm{Q}$ and $\mathrm{C} 5 \mathrm{X}$ signals up to an unknown constant.

\subsection{Difference of $D C B$ values between $Q$ and $X$ signals}

Since the code bias parameter is one-to-one correlated with the receiver clock offset, only the DCB value between signals can be determined. DCB values of GPS frequency L1-L5 and Galileo frequency E1-E5a, E1-E5b can be directly determined by the geometry-free pseudorange linear combination $(P I)$

$P I=P_{1}-P_{5}=I\left(1-\frac{f_{1}^{2}}{f_{5}^{2}}\right)+D C B_{r, 15}-D C B_{15}^{s}$

In our estimation, CODE MGEX orbit and global ionosphere map products are used to eliminate the ionospheric delays. A zero mean condition of all the satellite DCBs is imposed to separate satellite DCBs from those of the receivers. We use a network of 145 IGS Multi-GNSS stations (Fig. 1) based on 1 month of data from day of year (doy) 43-73 2019. Signals tracked by each receiver type are shown in Table 1 . The LEICA and SEPT network cluster is used to calculate DCB values of C1W-C5Q for GPS and C1C-C5Q, C1CC7Q for Galileo while the TRIMBLE and JAVAD network cluster is used to calculate the same types of DCB values that relate to X signal. For GPS L1 frequency, CODE monthly

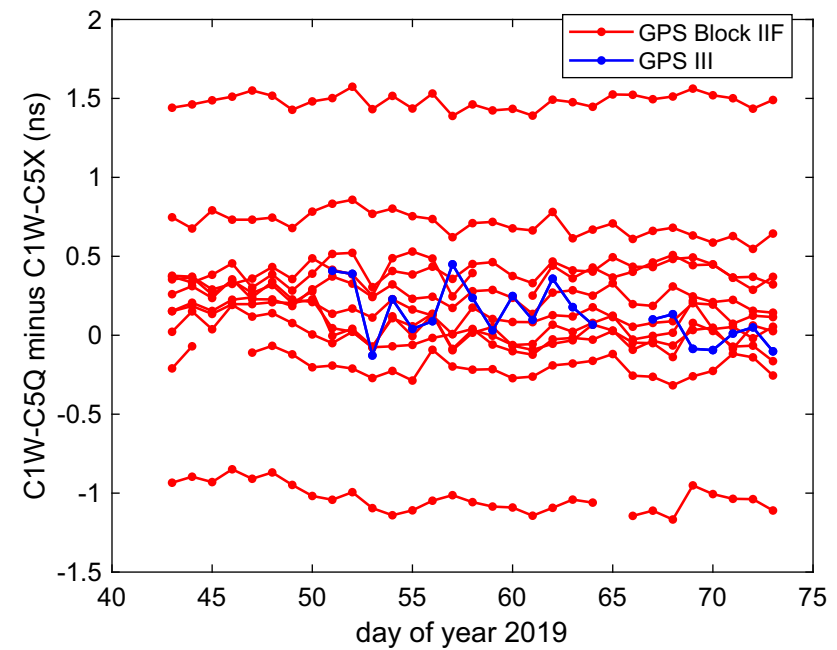

Fig. 2 Differences between DCB values of C1W-C5Q and C1W-C5X for GPS L1-L5 frequency, bias of G01 is removed from all the other satellites

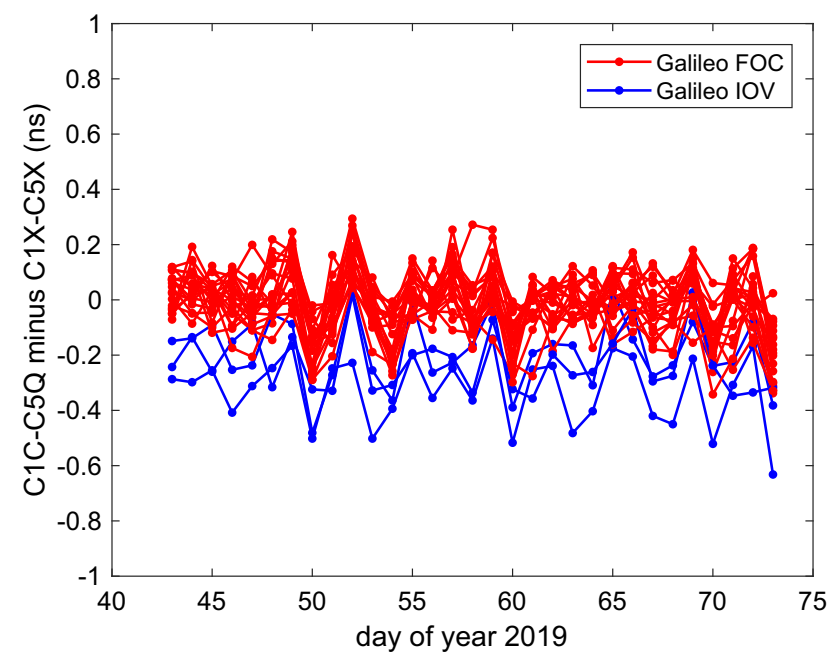

Fig. 3 Differences between DCB values of C1C-C5Q and C1X-C5X for Galileo E1-E5a frequency, bias of E01 is removed from all the other satellites

DCB products are used in advance to correct the $\mathrm{C} 1 \mathrm{C}$ signal to $\mathrm{C} 1 \mathrm{~W}$.

Figures 2, 3 and 4 show individual differences of DCB values between Q and X for GPS L1-L5 and Galileo E1-E5a, E1-E5b frequencies. The daily reference is removed from all the other satellites by differencing with the first satellite (G01 for GPS and E01 for Galileo). The difference of GPS Block IIF satellites can be as large as $1.5 \mathrm{~ns}$, which
Table 1 Signals tracked by different receiver types

\begin{tabular}{lllll}
\hline Receiver & GPS L1/L2 & GPS L1/L5 & Galileo E1/E5a & Galileo E1/E5b \\
\hline LEICA, SEPT & C1C/C1W, C2W & C1C/C1W, C5Q & C1C, C5Q & C1C, C7Q \\
TRIMBLE, JAVAD & C1C/C1W, C2W & C1C/C1W, C5X & C1X, C5X & C1X, C7X \\
\hline
\end{tabular}




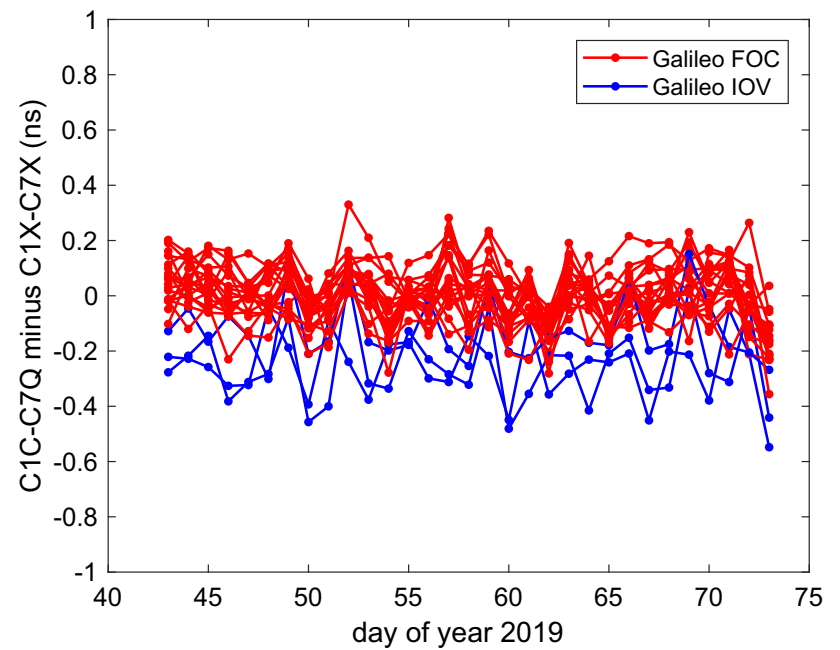

Fig. 4 Differences between DCB values of C1C-C7Q and C1X-C7X for Galileo E1-E5b frequency, bias of E01 is removed from all the other satellites

is about half of the wide-lane wavelength. Therefore, such differences cannot be ignored in the MelWub linear combination. The first GPS III satellite is not available everyday and cannot be tracked by all the stations. The daily DCB differences are close to zero, with a RMS value of 0.2 ns. Galileo E1-E5a and E1-E5b frequencies show smaller differences of DCB values between $\mathrm{Q}$ and $\mathrm{X}$ for all the satellites. It is also clearly displayed that Galileo In-Orbit Validation (IOV) satellites show 2-3 times larger difference values than the Full Operational Capability (FOC) satellites. RMS values of each type of satellite are given in Table 2. In general, differences of DCB values for Galileo signals will not cause large differences in satellite MelWub bias estimation.

\subsection{MelWub biases}

As shown in Eq. (3), satellite and receiver MelWub biases are one-to-one correlated, only the sum can be determined. To separate the estimates, we assume a zero mean condition of all the satellite MelWub biases. Since it is widely known that satellite MelWub biases vary very slow with time we estimate a daily MelWub bias for each satellite, but estimate an epochwise receiver bias to capture the potential variation caused by the receiver (Loyer et al. 2012). In addition, we select a reference ambiguity that has the longest observation time for one receiver in one session to cope with the singularity between ambiguity and receiver biases.

The Bernese GNSS Software 5.3 (Dach et al. 2015) is modified to support the estimation of satellite MelWub and narrow-lane biases as well as the PPP-AR application. The ambiguity resolution estimator is based on the Bernese SIGMA method (Dach et al. 2015). Code observations are smoothed by the phase observations shifted by the mean difference of code minus phase per observation arc. Code biases are not affected by the smoothing. Ambiguities are first estimated as real values, then, integer values of the ambiguities are resolved according to the real-valued estimates and the corresponding variances and covariances. GPS and Galileo signals are processed independently except that GPS L1/L5 and Galileo E1/E5a observations are processed together to compute GPS L1/L5 related results. Because there are only 12 GPS Block IIF and one GPS III satellites broadcasting L1/L5 signals during the experimental time periods. The joint processing with Galileo E1/E5a observations can enhance the receiver clock estimates in the later narrow-lane ambiguity resolution. For the combined processing, if we take GPS as the reference, an additional daily inter-system bias (ISB) parameter for the Galileo system should be estimated for each receiver. However, if we do not consider such an ISB in the MelWub linear combination, the effect in one station is absorbed by the wide-lane ambiguities. By forming single differences (one station and two satellites) between real-valued ambiguities of the same constellation the station specific ISB effect can be eliminated. As a consequence, the single differenced ambiguities can be resolved to their integer values even without the consideration of the ISB effect.

Satellite MelWub biases are estimated by the same two network clusters of the same time periods to investigate the difference between $\mathrm{Q}$ and X signals. For GPS L1/L2 frequencies, the potential $\mathrm{C} 1 \mathrm{C}$ signal of some stations is corrected to $\mathrm{C} 1 \mathrm{~W}$ in advance and signals in the two clusters are consistent. Therefore, normal equations of the two network clusters are combined to generate a final daily solution. However, we need to be careful that satellite MelWub biases determined by one network cluster might differ by integer wide-lane cycles
Table 2 RMS values of differences between DCB values, (ns)

\begin{tabular}{llll}
\hline Frequency & DCB difference & Maximum & Mean \\
\hline GPS Block IIF L1/L5 & (C1W-C5Q)-(C1W-C5X) & 1.50 & 0.45 \\
GPS III L1/L5 & (C1W-C5Q)-(C1W-C5X) & 0.20 & 0.20 \\
Galileo IOV E1/E5a & (C1C-C5Q)-(C1X-C5X) & 0.35 & 0.28 \\
Galileo FOC E1/E5a & (C1C-C5Q)-(C1X-C5X) & 0.14 & 0.11 \\
Galileo IOV E1/E5b & (C1C-C7Q)-(C1X-C7X) & 0.29 & 0.25 \\
Galileo FOC E1/E5b & $(\mathrm{C} 1 \mathrm{C}-\mathrm{C} 7 \mathrm{Q})-(\mathrm{C} 1 \mathrm{X}-\mathrm{C} 7 \mathrm{X})$ & 0.11 & 0.09
\end{tabular}



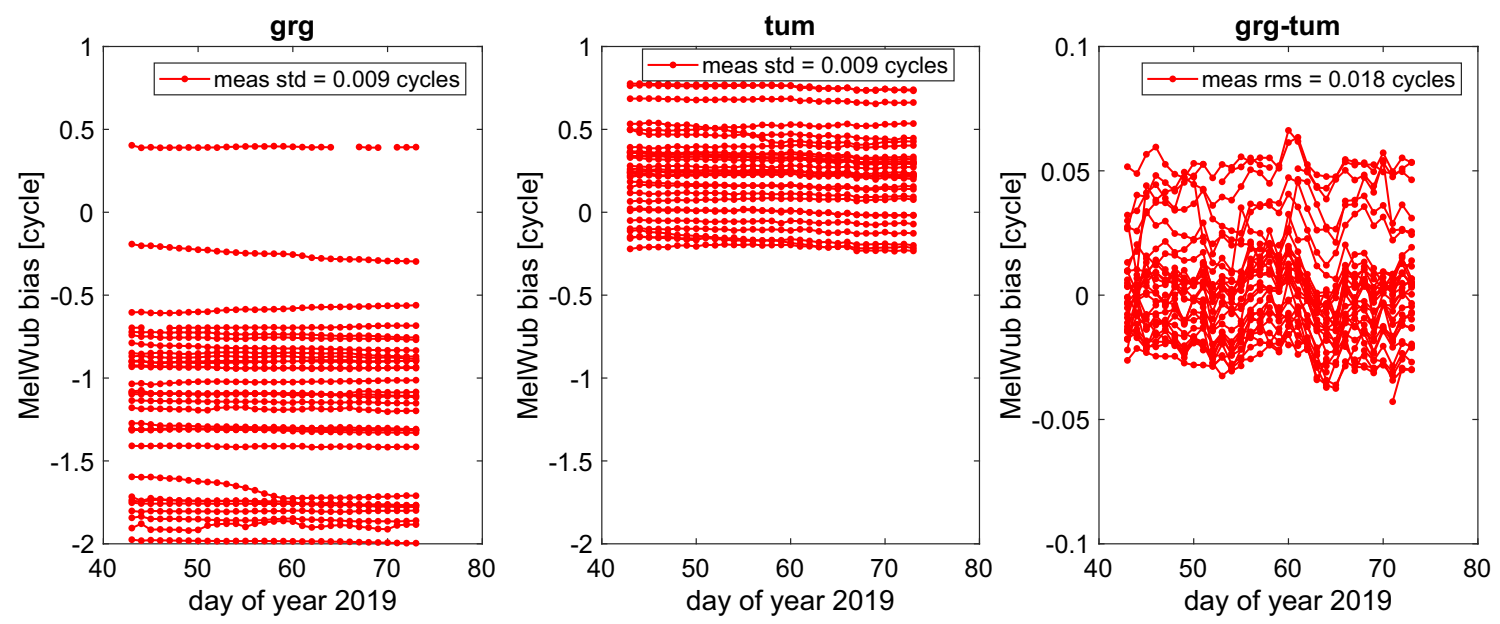

Fig. 5 CNES/CLS (grg), TUM (tum) daily GPS L1/L2 satellite MelWub biases and the difference (grg-tum) of these two, the reference (G01) and integer-cycle differences are eliminated from grg-tum
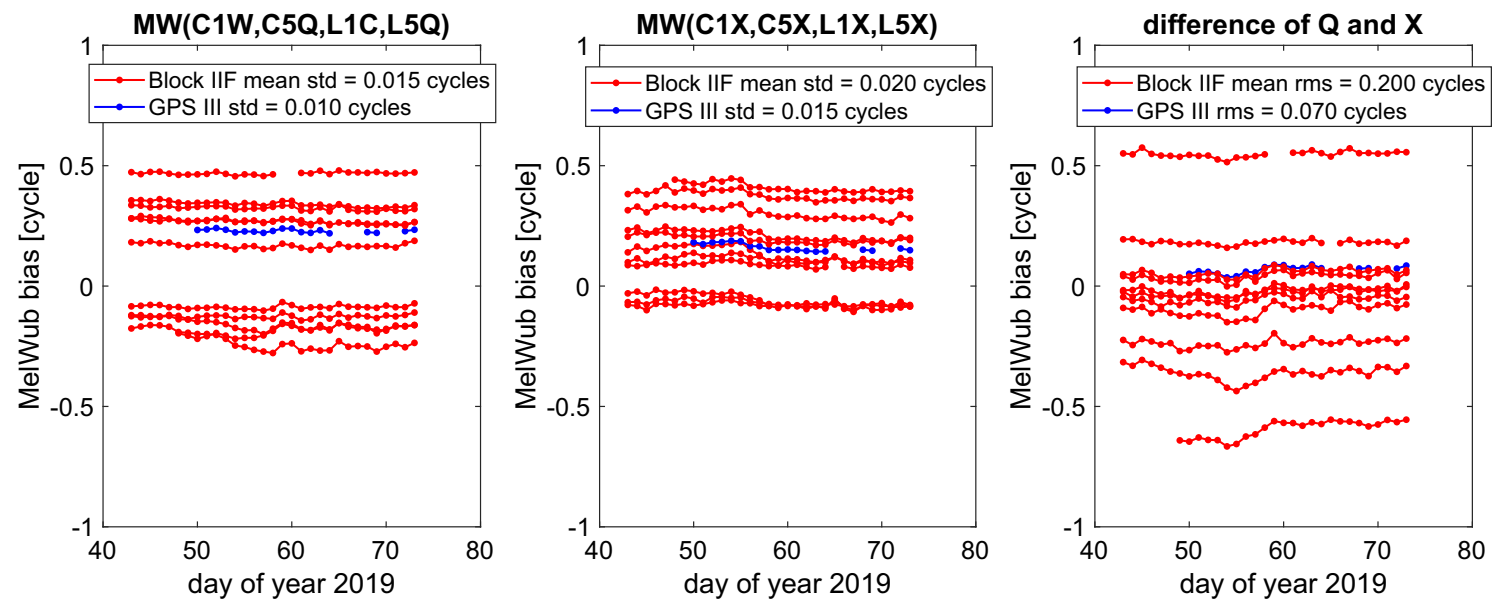

Fig. 6 GPS IIF and III satellite L1/L5 MelWub biases, left figure shows the estimation from Q signal, middle figure shows the estimation from X signal and the right figure displays the difference of these two by eliminating the reference satellite G01

from those computed by the other cluster. By making use of the stability of satellite MelWub bias, we constrain satellite MelWub biases in each cluster tightly to the estimates of the previous day. Therefore, we only need to make sure that satellite MelWub biases are correctly determined at the first day without any constraint. Since we use the same signals as CNES/CLS when estimating GPS L1/L2 MelWub biases we compare our estimates (TUM) to the CNES/CLS products. The left figure in Fig. 5 shows the CNES/CLS daily MelWub biases for all the GPS satellites, the middle figure shows our estimates and the right figure displays the difference of these two. The first GPS satellite G01 is eliminated from the comparison to remove the reference difference. The integer wide-lane cycles are removed as well from the difference since only the fractional parts are of interest. Both CNES/CLS and our products show small variations with time, the mean STD values of all the satellites over 1 month are both 0.009 wide-lane cycles. Of course, one reason is that both estimates are constrained tightly to the values from previous day. Also, these two products are consistent, the mean RMS value of differences is 0.018 wide-lane cycles.

For GPS L1/L5, Galileo E1/E5a and Galileo E1/E5b frequencies, performances and differences of satellite MelWub biases from the two network clusters are shown in Figs. 6, 7 and 8. If we ignore the differences in the network satellite MelWub biases computed by the two network clusters should be identical. Then, the displayed differences can be attributed to the differences between signal Q and X. We find that the maximum difference of GPS Block IIF satellites is more than 0.5 wide-lane cycles. We cannot mix these two signals together in the MelWub bias estimation. The differences of the GPS III satellite are not significant, with a RMS 

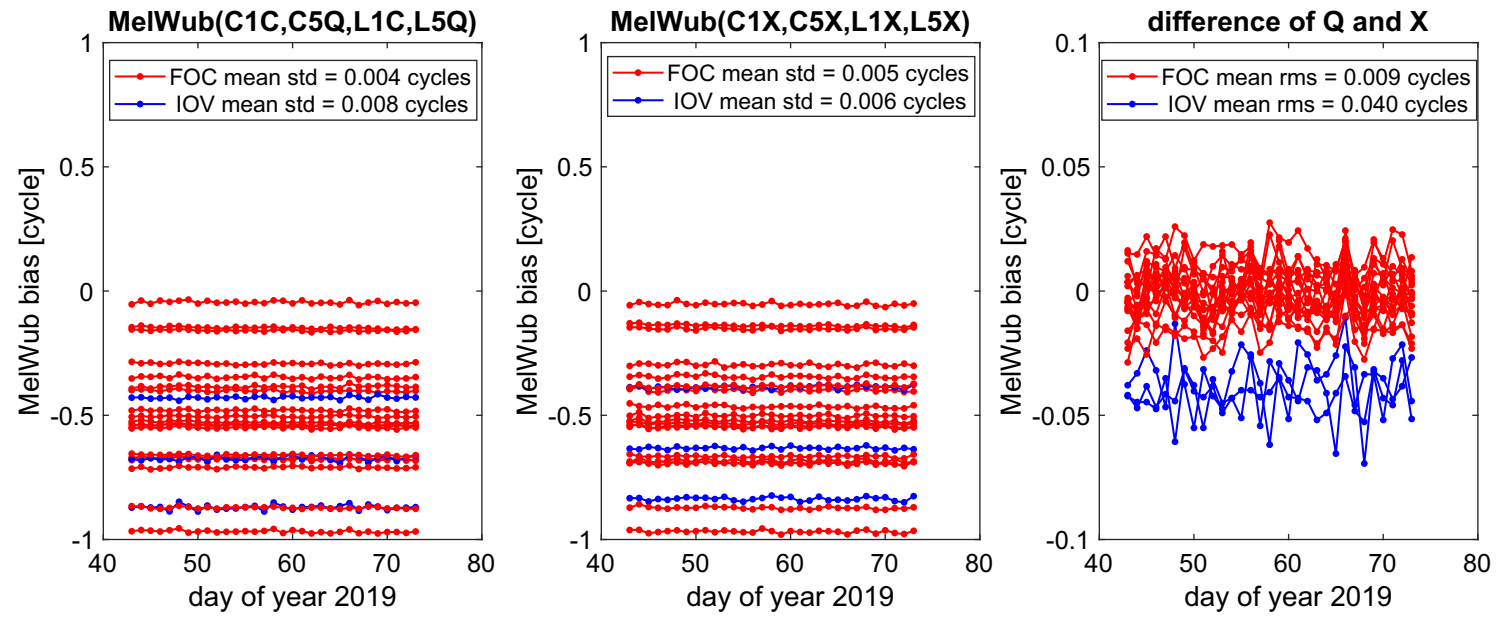

Fig. 7 Galileo satellite E1/E5a MelWub biases, left figure shows the estimation from Q signal, middle figure shows the estimation from X signal and the right figure displays the difference of these two by eliminating the reference satellite E01
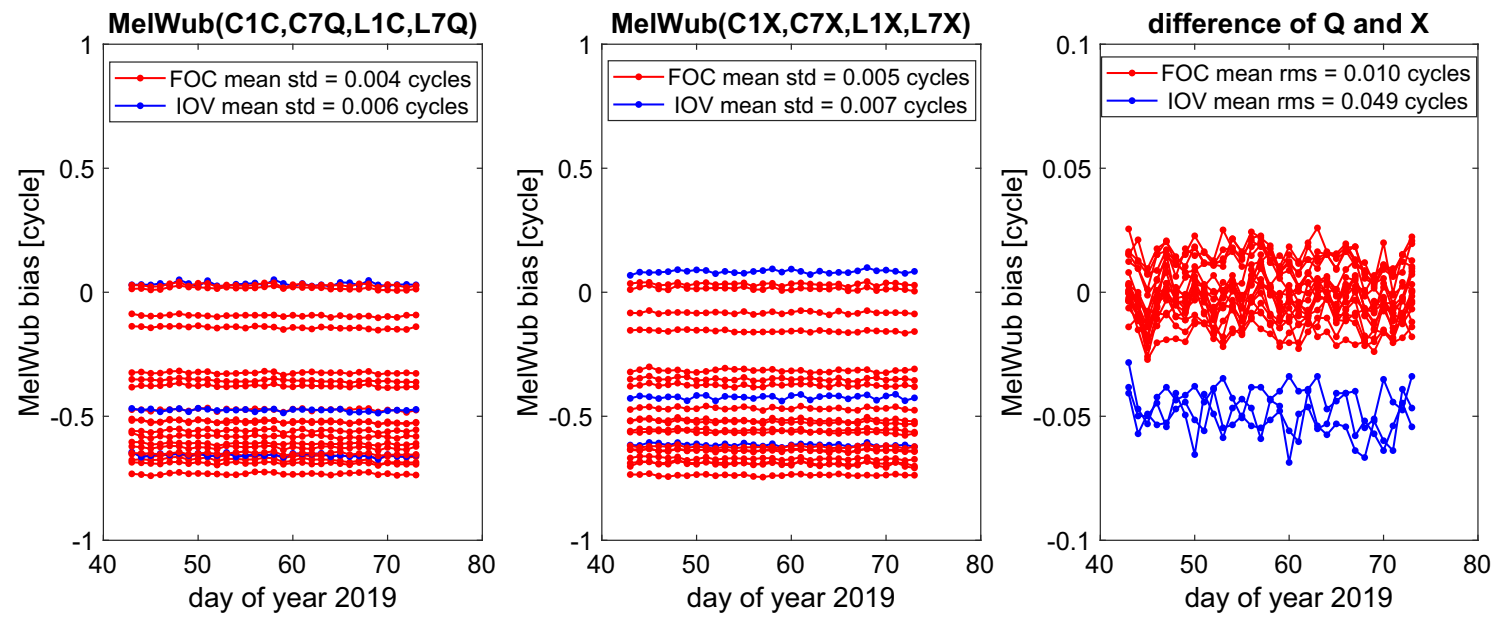

Fig. 8 Galileo satellite E1/E5b MelWub biases, left figure shows the estimation from Q signal, middle figure shows the estimation from X signal and the right figure displays the difference of these two by eliminating the reference satellite E01

value of 0.07 wide-lane cycles. All the Galileo satellite MelWub biases are more constant over time than those of GPS L1/L2 estimates. Both Galileo E1/E5a and E1/E5b frequencies show small differences of MelWub bias between signal $\mathrm{Q}$ and X. In particular, the mean RMS value of differences for Galileo FOC satellites is 0.01 wide-lane cycles. Considering the slightly larger differences in DCB values for Galileo IOV satellites, we observe larger differences in MelWub biases as well. Nevertheless, the mean RMS value for Galileo IOV satellites is smaller than 0.05 wide-lane cycles. As a result, we can stack normal equations of these two network clusters for Galileo E1/E5a and E1/E5b frequencies, respectively, to generate combined $(\mathrm{Q}$ and $\mathrm{X})$ satellite MelWub bias products.

\section{Satellite orbits, integer clocks and narrow-lane biases}

Once the wide-lane ambiguity is known, Eq. (4) can be conducted by introducing the wide-lane ambiguity into Eq. (2). In this section, we introduce our procedures in computing satellite orbits, integer clock offsets and narrow-lane biases. To ensure a higher ambiguity fixing rate and to shorten the processing time, we use only phase measurements for ambiguity resolution and divide the whole station network into two network clusters.

\subsection{Procedures}

First, we use only the phase equation to determine satellite orbit and station related parameters (coordinates and tropospheric delays), as shown 


$$
\begin{aligned}
L_{r, I F}^{s}= & \rho_{r, I F}^{s}+c\left(\widehat{d t}_{r}-\widehat{d t}^{s}\right)+T_{r}^{s} \\
& +\lambda_{n l}\left(N_{r, 1}^{s}+\frac{\lambda_{w l}}{\lambda_{2}} N_{r, w l}^{s}\right)+\varepsilon_{r, I F}^{s}
\end{aligned}
$$

where $\widehat{d t}_{r}$ and $\widehat{d t}^{s}$ aggregate respective clock offset and phase bias (Loyer et al. 2012). The same two network clusters as shown in Fig. 1 are used to resolve ambiguities independently. The ambiguity that has the longest observation time in the session for each station is chosen as the reference ambiguity. All the reference ambiguities in the network are fixed to the closest integer values to cope with the singularity between ambiguities and clock offsets. As a result, clock offsets in this step are determined by phase ambiguities. We cannot combine the clock estimates from these two network clusters since they may differ by integer narrow-lane cycles. However, since phase clock offsets are pre-eliminated from the normal equation every epoch the combination of orbit parameters is not affected. Then, we stack the normal equations from these two clusters and generate daily ambiguity fixed satellite orbit products.

Second, we estimate real-valued satellite clock products while fixing satellite orbits and station related parameters to the estimates in step 1. Both code and phase measurements (as shown in Eq. (7)) are used to align satellite clock estimates to the IGS clocks. All the stations are processed in a single network since ambiguity resolution is not needed in this step.

$$
\begin{aligned}
P_{r, I F}^{s}= & \widehat{\rho}_{r, I F}^{s}+c\left(d t_{r}-d t^{s}\right)+c\left(d_{r, I F}-d_{I F}^{s}\right)+e_{r, I F}^{s} \\
L_{r, I F}^{s}= & \widehat{\rho}_{r, I F}^{s}+c\left(\widehat{d t}_{r}-d t^{s}\right)+\widehat{I F}\left(\lambda_{1} N_{r, 1}^{s}, \lambda_{2} N_{r, 2}^{s}\right) \\
& +\varepsilon_{r, I F}^{s}
\end{aligned}
$$

where $\widehat{\rho}_{r, I F}^{s}$ represents the geometric distance computed by the known satellite orbits and station coordinates. The $I F$ combined code biases $\left(d_{r, I F}\right.$ and $\left.d_{I F}^{S}\right)$ of all the dualfrequency types are assumed to be zero according to the IGS clock datum definition. $\widehat{I F}\left(\lambda_{1} N_{r, 1}^{s}, \lambda_{2} N_{r, 2}^{s}\right)$ contains satellite phase biases. It may be noted that the code receiver clock offset $d t_{r}$ differs from the phase receiver clock offset $\widehat{d t}_{r}$. However, the difference is within a narrow-lane cycle and can be neglected except for time transfer applications. We can simply estimate one set of receiver clock offset for both code and phase measurements every epoch.

Third, we estimate daily constant satellite narrow-lane biases while fixing satellite orbit, clock and station related parameters to the known estimates in step 1 and 2 . To ensure high narrow-lane fixing rate, we use only phase measurements in this step, as shown in Eq. (8) by eliminating all the known terms from Eq. (6).

$$
\begin{aligned}
L_{r, I F}^{s}-\lambda_{n l} \frac{\lambda_{w l}}{\lambda_{2}} N_{r, w l}^{s}= & \widehat{\rho}_{r, I F}^{s}+c \widehat{d t}_{r} \\
& +\lambda_{n l}\left(N_{r, 1}^{s}-b_{n l}^{s}\right)+\varepsilon_{r, I F}^{s}
\end{aligned}
$$

where $b_{n l}^{s}$ is the satellite narrow-lane bias consisting of $b_{I F}^{s}$ and the bias introduced by the integer wide-lane ambiguity. A zero mean condition of all the satellite narrow-lane biases is imposed to cope with the singularity between satellite narrow-lane biases and receiver clock offsets. Ambiguities are resolved independently in the same two network clusters as in step 1 . The potential integer narrow-lane cycles in satellite narrow-lane biases from different network clusters are aligned to their fractional parts before the stacking of normal equations. All the resolved ambiguities are saved consistently with respect to the final satellite narrow-lane biases. In this step, the saved ambiguities of one station can cause a constant shift in the receiver clock offsets.

Fourth, we estimate integer satellite clock products using both code and phase measurements of the whole station network. Satellite orbit, narrow-lane bias and station related parameters are fixed to the known values determined by step 1-3. The observation equation is shown in Eq. (9) where the code bias terms are not given since they are assumed to be zero.

$$
\begin{aligned}
& P_{r, I F}^{s}=\widehat{\rho}_{r, I F}^{s}+c\left(d t_{r}-d t^{s}\right)+e_{r, I F}^{s} \\
& L_{r, I F}^{s}-\lambda_{n l} \frac{\lambda_{w l}}{\lambda_{2}} N_{r, w l}^{s}=\widehat{\rho}_{r, I F}^{s}+c\left(\widehat{d t}_{r}-d t^{s}\right) \\
& \quad+\lambda_{n l} N_{r, 1}^{s}+\varepsilon_{r, I F}^{s}
\end{aligned}
$$

Satellite clock offsets determined by step 2 are taken as a priori values in this step. A zero mean condition of all the satellite clock offsets every epoch is imposed to separate satellite clock offsets from those of the receivers. All the resolved ambiguities in step 3 are taken as known in this step and phase equation in Eq. (9) is used to fix more ambiguities in the single combined network. After fixing all the ambiguities, code observations are introduced as well to estimate integer clock products. Finally, step 1 to step 4 can be iterated once to see whether more ambiguities can be fixed. The diagram describing the whole procedure is shown in Fig. 9.

\subsection{Products assessment}

We process the same 1 month of data as that in Sect. 3. Settings and orbit models are given in Table 3 . The observation sampling is $5 \mathrm{~min}$. We extract the middle day of the 3-dayarc orbit as the final daily solution. Earth rotation parameters (ERPs) are fixed to values in the Bulletin A file. The new Empirical CODE orbit model (ECOM2) is used for GPS satellites without any other a priori model while an a priori box-wing (BW) model is jointly used with the classical ECOM model for Galileo satellites (Beutler et al. 1994; Arnold et al. 2015; Duan et al. 2019). Phase center offset and variation (PCO and PCV) are fixed to the values in the standard IGS14 antex file. Stochastic pulses are considered 


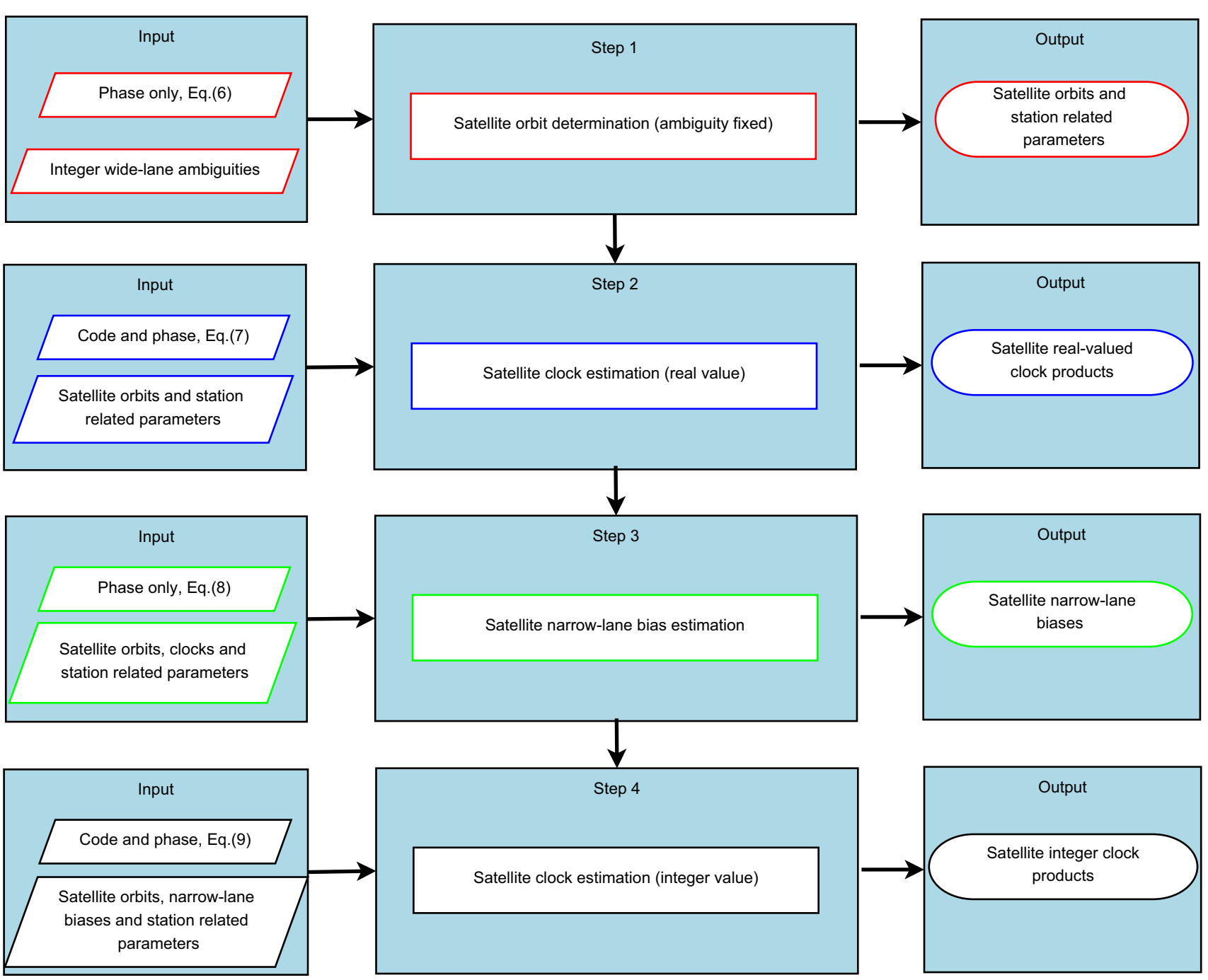

Fig. 9 Diagram of satellite orbit, integer clock and narrow-lane products estimation

for all the GPS satellites every $12 \mathrm{~h}$ while for Galileo satellites they are not applied. Station related parameters that are calculated from GPS L1/L2 observations are fixed as known in the following processing for GPS L1/L5, Galileo E1/E5a and Galileo E1/E5b observations.

We calculate orbit differences in radial, along-track and cross-track components at the day boundaries between consecutive arcs and define such differences as orbit misclosures. Table 4 shows the mean RMS values of orbit misclosures with and without ambiguity resolution for individual frequencies. It is clear that the ambiguity fixed solutions perform better than the float solutions for both GPS and Galileo satellites by using all frequencies. In particular, the improvement in along-track component is more than a factor of two. It is also noticed that orbit misclosures of the ambiguity fixed Galileo satellites are almost at the same level as those of GPS. We also compare our estimates to the external CODE MGEX orbits, as shown in Table 5. Same as in Table 4, ambiguity fixed solutions reduce the mean RMS values of 3D orbit differences by about a factor of two. In addition, we analyze satellite laser ranging (SLR) (Pearlman et al. 2002) residuals of both types of Galileo satellite orbits, as shown in Fig. 10. We find that the ambiguity fixed results show almost the same SLR residuals as the float solutions and there is no dependency of SLR residuals on the $\beta$ angle (Sun elevation above the orbital plane).

The IGS GPS and Galileo satellite clock products are based on L1/L2 and E1/E5a frequencies, respectively. We compare our clock estimates of the same frequency to the CODE MGEX satellite clock products since they provide ambiguity fixed clocks for the same time period. Figure 11 illustrates the daily mean STD value of clock differences. For both GPS and Galileo satellites, the mean STD values are reduced by about a factor of two when fixing ambiguity parameters to the integer values. It may be noted that the bottom figure of Fig. 11 exhibits a clear shift of STD values over 
Table 3 Settings and orbital models

\begin{tabular}{ll}
\hline Items & Value \\
\hline Software & Bernese 5.3 modified (Dach et al. 2015) \\
Observations & Zero-difference ionosphere-free \\
Frequencies & L1/L2, L1/L5, E1/E5a, E1/E5b \\
Data sampling & 5 min \\
Orbit arc & Middle day of 3-day-arc \\
PCO and PCV & IGS14 ANTEX \\
ERP & IERS bulletin A \\
Solar radiation pressure & GPS: ECOM2 (Arnold et al. 2015) \\
& Galileo: ECOM + Box-wing \\
Earth albedo & Considered (Rodriguez-Solano et al. 2012) \\
Antenna thrust & Considered (Steigenberger et al. 2018) \\
$\begin{array}{l}\text { Ambiguity resolu- } \\
\text { tion estimator }\end{array}$ & SIGMA (Dach et al. 2015) \\
Stochastic pulses & GPS: yes \\
& Galileo: no \\
\hline &
\end{tabular}

Table 4 Mean RMS of orbit misclosures, R means radial component, A denotes along-track component, $\mathrm{C}$ means cross-track component (unit: $\mathrm{cm})$

\begin{tabular}{llllllll}
\hline Frequency & \multicolumn{1}{l}{ Float } & & & & \multicolumn{3}{c}{ Ambiguity fixed } \\
\cline { 2 - 3 } & $\mathrm{R}$ & $\mathrm{A}$ & $\mathrm{C}$ & & $\mathrm{R}$ & $\mathrm{A}$ & $\mathrm{C}$ \\
\hline $\mathrm{L} 1 / \mathrm{L} 2$ & 0.3 & 2.1 & 1.3 & & 0.3 & 0.8 & 0.7 \\
$\mathrm{~L} 1 / \mathrm{L} 5$ & 0.3 & 2.5 & 1.3 & & 0.3 & 1.1 & 0.8 \\
E1/E5a & 0.5 & 2.8 & 1.6 & & 0.5 & 1.0 & 1.2 \\
E1/E5b & 0.6 & 2.4 & 1.6 & & 0.5 & 1.1 & 1.3 \\
\hline
\end{tabular}

Table 5 Mean RMS of orbit difference by comparing with CODE MGEX orbits, $\mathrm{R}$ means radial component, A denotes along-track component, $\mathrm{C}$ means cross-track component (unit: $\mathrm{cm}$ )

\begin{tabular}{llllllll}
\hline Frequency & \multicolumn{1}{l}{ Float } & & & & \multicolumn{3}{c}{ Ambiguity fixed } \\
\cline { 2 - 3 } & $\mathrm{R}$ & $\mathrm{A}$ & $\mathrm{C}$ & & $\mathrm{R}$ & $\mathrm{A}$ & $\mathrm{C}$ \\
\hline L1/L2 & 1.3 & 3.8 & 2.5 & & 0.8 & 1.6 & 1.5 \\
L1/L5 & 1.3 & 3.3 & 2.7 & & 1.0 & 1.8 & 1.7 \\
E1/E5a & 2.3 & 3.3 & 2.9 & 2.1 & 2.2 & 1.7 \\
E1/E5b & 2.3 & 3.4 & 3.1 & & 2.1 & 2.3 & 1.8 \\
\hline
\end{tabular}

time. This is because that $\beta$ angles of satellites in one orbit plane are getting close to zero. The potential reason might be due to the different usage of solar radiation pressure (SRP) models.

\subsection{Differences between dual-frequency types}

In the IGS14 antex file, PCO and PCV values of Galileo satellites are calibrated for all the frequencies while those of GPS L5 frequency are not yet given. Receiver PCO and PCV

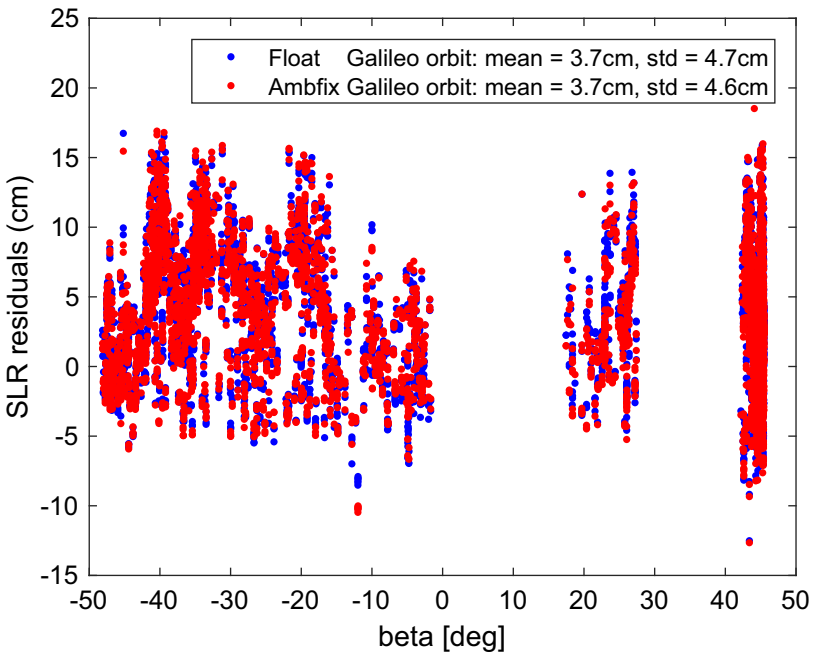

Fig. 10 SLR residuals of float (blue) and ambiguity fixed (red) Galileo satellite orbits

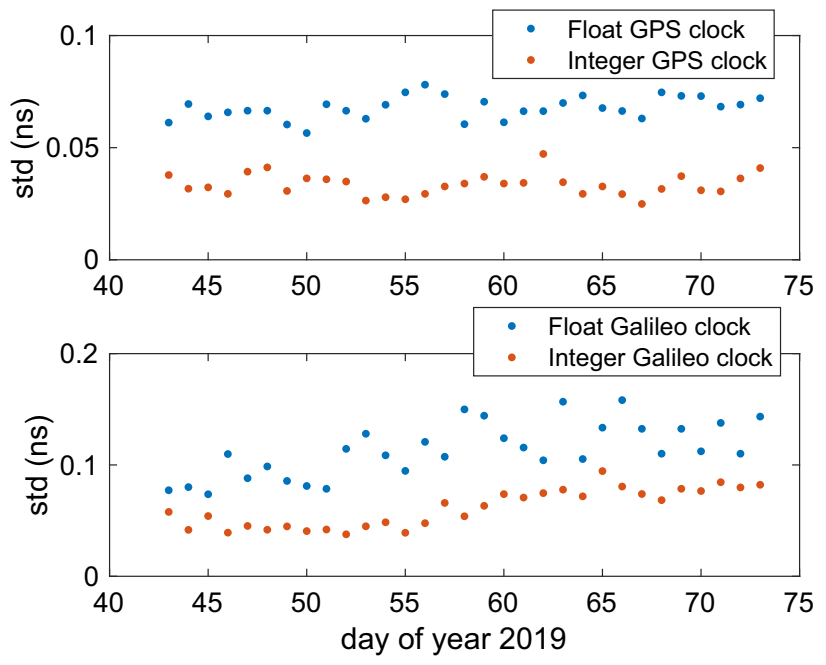

Fig. 11 Mean std values of satellite clock difference between our estimates and CODE MGEX products, the top figure shows GPS L1/L2 clocks while the bottom figure shows Galileo E1/E5a clocks, blue color represents float solution, red color denotes ambiguity fixed solution

values are only available for GPS L1 and L2 frequencies. Thus, we assume that PCO and PCV values of GPS satellites on frequency L5 are the same as those on frequency L2. PCO and PCV values of receivers on GPS L1/L2 are copied for GPS L1/L5, Galileo E1/E5a and E1/E5b. To investigate the impact, we compare GPS and Galileo orbit products determined by different dual-frequency types. We find that the mean RMS of orbit differences between GPS L1/L2 and $\mathrm{L} 1 / \mathrm{L} 5$ is $0.7,1.1$ and $0.8 \mathrm{~cm}$ in radial, along- and cross-track components. For Galileo satellites, orbit differences between E1/E5a and E1/E5b are smaller, with a mean RMS of 0.3, 0.8 and $0.6 \mathrm{~cm}$ in radial, along- and cross-track components. It 

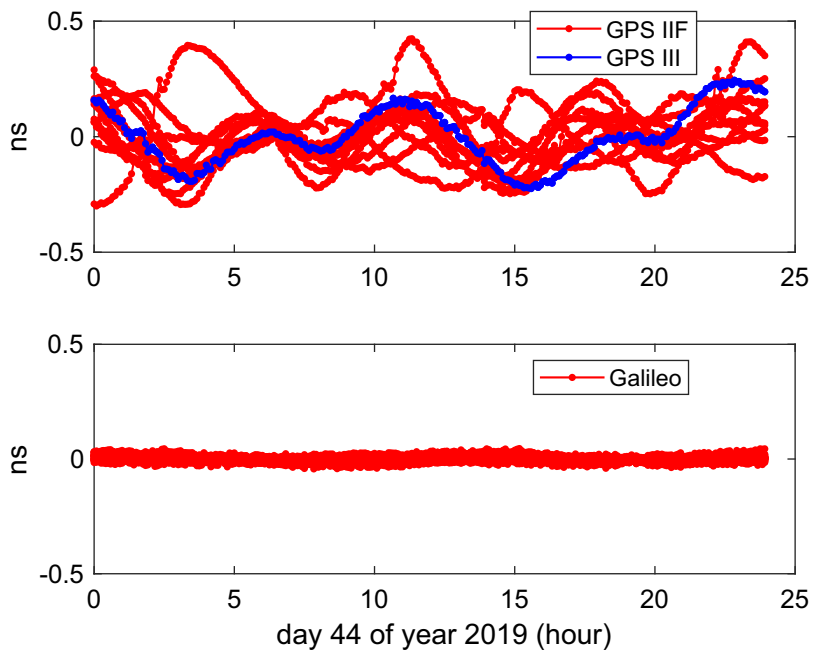

Fig. 12 Clock difference of 1 day between L1/L2 and L1/L5 estimates for GPS satellites (top) and between E1/E5a and E1/E5b estimates for Galileo satellites (bottom), the mean difference of each satellite is removed

is also mentioned that the station network of GPS L1/L2 and L1/L5, Galileo E1/E5a and E1/E5b is not identical since not all the stations track the frequency L5 or E5b. As a consequence, orbit differences between different dual-frequency types are not significant, especially for Galileo satellites.

Differences of GPS satellite clock offsets between frequency L1/L2 and L1/L5 are confirmed due to the inconsistency of carrier phase measurements (Montenbruck et al. 2012). Similarly, we perform an additional experiment with Galileo E1/E5a and E1/E5b measurements. Figure 12 shows clock differences of 1 day between L1/L2 and L1/L5 estimates for GPS satellites (top) and between E1/E5a and E1/E5b estimates for Galileo satellites (bottom). The mean offset of clock differences is eliminated from each satellite. It is clear that clock differences between GPS L1/L2 and L1/L5 vary periodically within 1 day, which just repeats the results in the citation. The peak-to-peak amplitudes start from -0.3 to $0.4 \mathrm{~ns}$, and the mean RMS of clock difference over 1 month is $0.1 \mathrm{~ns}$. For Galileo satellites, the clock differences between the two types of dual-frequencies are very close to zero. All the differences are smaller than $0.04 \mathrm{~ns}$ and the mean RMS over 1 month is $0.015 \mathrm{~ns}$, which is at the same level as the noise of carrier phase measurement in the ionosphere-free linear combination. Therefore, phase users can apply Galileo E1/E5a (same as in MGEX products) satellite clock products for the E1/E5b measurements. This is also very helpful for the triple frequency applications. However, for the pseudorange-only users, the mean difference of each satellite cannot be absorbed by ambiguity parameters and therefore needs to be considered.

\section{Applications}

We have estimated satellite orbit, clock, daily MelWub bias and daily narrow-lane bias products for GPS L1/L2, L1/L5 and Galileo E1/E5a, E1/E5b frequencies, respectively. 30second sampling satellite clock products are generated based on the 5-min clock products using an efficient approach (Bock et al. 2009). To assess these products, we first introduce them into ground-station PPP application. The observation sampling is $30 \mathrm{~s}$. Since there are not many GPS satellites broadcasting L1/L5 signals the corresponding applications are not investigated in this contribution. We use five stations that are not employed in the product estimation, as shown in Table 6. Data time interval is the same 1 month as in Sects. 3 and 4. Satellite products determined by different frequencies (L1/L2, E1/E5a and E1/E5b) are applied in the PPP-AR application, respectively. Table 7 shows the ambiguity fixing rates and the mean daily repeatability of PPP solutions. The fixing rates of Galileo satellites are in general slightly higher than that for GPS satellites. For all the three types of frequencies, the ambiguity fixed PPP solutions improve the repeatability of station coordinates by about $10 \%$ in north and up directions while about $30 \%$ in the east direction. Galileo satellites show similar performance as GPS satellites in the PPP-AR application.

Then, we apply GPS L1/L2 products for Sentinel-3A (Peter et al. 2017; Montenbruck et al. 2018; Duan and Hugentobler 2019) satellite orbit determination. Sampling of GPS observations is $30 \mathrm{~s}$. We assess both reduced-dynamic and kinematic orbit solutions. Figure 13 shows the SLR residuals of reduced-dynamic orbit solutions. The STD value is reduced by about $10 \%$ by comparing ambiguity fixed solutions to float valued solutions. Figure 14 shows the performance of kinematic orbits with respect to the reduceddynamic solutions. The $3 \mathrm{D}\left(3 D=\sqrt{\Delta x^{2}+\Delta y^{2}+\Delta z^{2}}\right)$ RMS of orbit differences reduces by about a factor of two when fixing ambiguities to integer values. Both ground station PPP application and Sentinel satellite orbit determination demonstrate that our GPS and Galileo satellite products perform fairly well in the zero-difference ambiguity resolution.

\section{Summary and conclusions}

Different than fixing double-difference ambiguities, ambiguity resolution of a single receiver needs dedicated satellite clock and bias products. In this contribution we present efficient procedures to estimate ambiguity fixed satellite orbit, integer clock, daily MelWub and narrow-lane bias products for both GPS and Galileo satellites.

First, we focus on the code bias differences between pilotonly signal Q and pilot-data signal X since satellite MelWub 
Table 6 Station information in PPP test

\begin{tabular}{lll}
\hline Station & Receiver type & Observations \\
\hline BSHM & JAVAD & C1W, L1C, C2W, L2W, C1X, L1X, C5X, L5X, C7X, L5X \\
KIRU & SPET & C1W, L1C, C2W, L2W, C1C, L1Q, C5Q, L5Q, C7Q, L7Q \\
TSK2 & TRIMBLE & C1W, L1C, C2W, L2W, C1X, L1X, C5X, L5X, C7X, L5X \\
USN8 & SEPT & C1W, L1C, C2W, L2W, C1C, L1Q, C5Q, L5Q, C7Q, L7Q \\
WTZS & JAVAD & C1W, L1C, C2W, L2W, C1X, L1X, C5X, L5X, C7X, L5X \\
\hline
\end{tabular}

Table 7 Wide-lane (WL) and narrow-lane (NL) ambiguity fixing rates and mean daily repeatability of all the stations over 1 month, Q means stations tracking signal $\mathrm{Q}, \mathrm{X}$ denotes stations tracking signal $\mathrm{X}$ (unit: $\mathrm{mm})$

\begin{tabular}{|c|c|c|c|c|c|c|c|c|}
\hline \multirow[t]{2}{*}{ Satellite and frequency } & \multicolumn{2}{|c|}{ Ambiguity fixing rate } & \multicolumn{3}{|l|}{ Float } & \multicolumn{3}{|c|}{ Ambiguity fixed } \\
\hline & WL (\%) & NL (\%) & North & East & Up & North & East & Up \\
\hline GPS L1/L2 & 92.0 & 88.8 & 2.1 & 3.2 & 5.3 & 1.9 & 2.4 & 4.8 \\
\hline Galileo E1/E5a (Q) & 94.2 & 90.9 & 2.2 & 3.3 & 5.2 & 2.0 & 2.4 & 4.7 \\
\hline Galileo E1/E5a (X) & 96.0 & 92.4 & 2.1 & 3.0 & 4.8 & 2.0 & 2.1 & 4.3 \\
\hline Galileo E1/E5b (Q) & 94.2 & 90.9 & 2.3 & 3.4 & 5.5 & 2.0 & 2.5 & 5.0 \\
\hline Galileo E1/E5b (X) & 94.5 & 90.8 & 2.1 & 3.2 & 5.0 & 2.1 & 2.4 & 4.6 \\
\hline
\end{tabular}

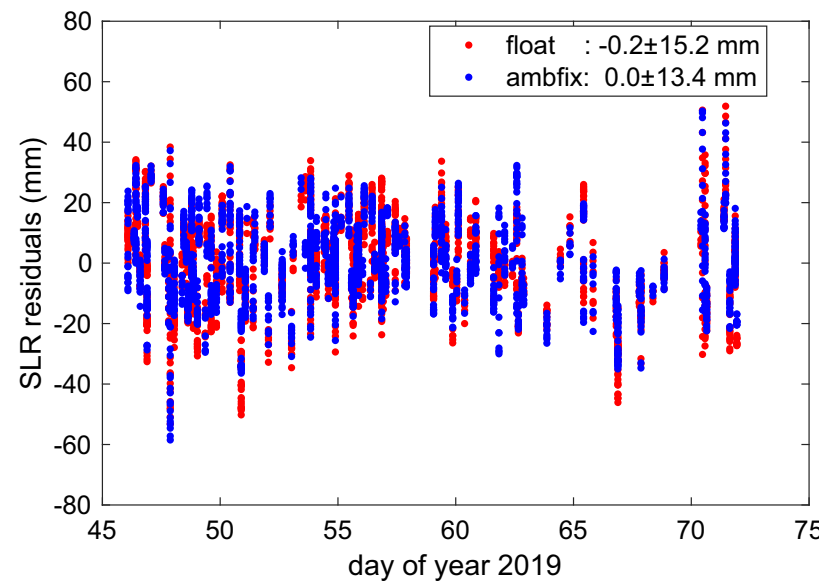

Fig. 13 SLR residuals of Sentine-3A reduced-dynamic satellite orbits by using our GPS products, red color represents float solution, blue color denotes ambiguity fixed solution

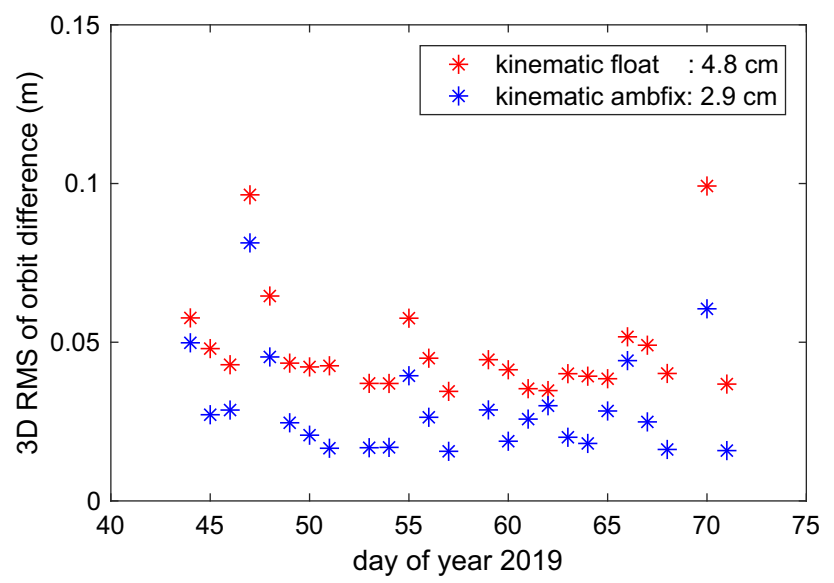

Fig. 14 3D daily RMS of orbit difference between kinematic and reduced-dynamic Sentinel-3A satellite orbit, red color denotes the float solution while blue color denotes the ambiguity fixed solution biases aggregate various code and phase biases. To investigate the detail, we set up two network clusters tracking Q and X signal, respectively. Results demonstrate that unlike phase measurements, GPS C5Q and C5X signals cannot be mixed in a network to estimate common satellite MelWub biases due to the large differences of code biases. Whereas, the mixing of C5Q and C5X is possible for Galileo satellites. The RMS of MelWub bias differences determined by $Q$ and $\mathrm{X}$ network is 0.01 wide-lane cycles, which is negligible. So, we do not need to consider the difference between $\mathrm{Q}$ and $\mathrm{X}$ signals in Galileo satellite MelWub bias estimation.

Then, we estimate satellite orbit, integer clock and narrowlane bias products. Orbit misclosures and orbit differences with respect to the CODE Final orbits show that ambiguity resolution improves the precision of GPS and Galileo satellite orbits by about a factor of two, especially in the along-track direction. The integer satellite clock products are confirmed to be more consistent with the CODE clock products. In addition, we compare orbit and clock solutions based on individual dual-frequency types for GPS and Galileo satellites, respectively. Clock differences between GPS L1/L2 and L1/L5 estimates show clear periodical variations, with a peak-to-peak amplitude of $0.7 \mathrm{~ns}$ after removing the daily mean difference of each satellite. However, the maximum clock difference between Galileo E1/E5a and E1/E5b estimates after removing the mean value is $0.04 \mathrm{~ns}$ and the mean RMS of differences is $0.015 \mathrm{~ns}$, which is at the same level as the noise of carrier phase measurement in the ionospherefree linear combination. So, phase measurement users can apply MGEX Galileo satellite clock products (determined by E1/E5a) in the applications using E1/E5b phase measurements.

Finally, we introduce our GPS and Galileo products into precise applications. We take five stations that are not used in 
the product estimation and assess performances of PPP-AR. The mean repeatability of station coordinates improves by about $10 \%$ in north and up directions while by about $30 \%$ in the east direction by fixing ambiguities to integer values. We also introduce our GPS L1/L2 products into Sentinel-3A satellite orbit determination. The STD value of SLR residuals is reduced by about $10 \%$ for the reduced-dynamic orbits by fixing ambiguity parameters to integer values. Moreover, the ambiguity fixed kinematic orbit solutions are two times more consistent with the reduced-dynamic solutions.

Acknowledgements This research is based on the analysis of GPS and Galileo observations provided by the IGS. We compare our estimates to the CODE and CNES/CLS analysis centers. The effort of all the IGS agencies and organizations as well as all the analysis centers is acknowledged. Sentinel-3A data used in this study comes from the European Commission and the European Space Agency (ESA) as part of the Copernicus program. We appreciate the continued technical support from GMV and the Copernicus Precise Orbit Determination (CPOD) working group. Satellite laser ranging data of Galileo and Sentinel-3A satellite is taken from http://cddis.gsfc.nasa.gov, we would like to thank to International Satellite Laser Ranging Service (ILRS) as well as the effort of all respective stations operators. We would like to acknowledge Tim Springer and two other anonymous reviewers for their very helpful comments for this paper.

Author Contributions BD and UH proposed the general idea of this contribution, designed the experiments, analyzed the results and wrote the paper. BD adopted the Bernese software. IS contributed to the attitude model of GPS and Galileo satellites. NW contributed to the satellite DCB calculation.

Funding Open Access funding enabled and organized by Projekt DEAL.

Data availability GNSS and SLR data used are publicly available from International GNSS Service and International Satellite Laser Ranging Service from the respective data centers, Sentinel-3 data are publicly available at ESA (European Space Agency) open access data Hub.

\section{Declaration}

Conflict of interest The authors confirm that there are no conflicts of interest and no competing interests.

Code availability Calculations are based on the Bernese GNSS software (license available, University of Bern) and its specific modifications by the authors (not public).

Open Access This article is licensed under a Creative Commons Attribution 4.0 International License, which permits use, sharing, adaptation, distribution and reproduction in any medium or format, as long as you give appropriate credit to the original author(s) and the source, provide a link to the Creative Commons licence, and indicate if changes were made. The images or other third party material in this article are included in the article's Creative Commons licence, unless indicated otherwise in a credit line to the material. If material is not included in the article's Creative Commons licence and your intended use is not permitted by statutory regulation or exceeds the permitted use, you will need to obtain permission directly from the copy- right holder. To view a copy of this licence, visit http://creativecomm ons.org/licenses/by/4.0/.

\section{References}

Arnold D, Meindl M, Beutler G, Dach R, Schaer S, Lutz S, Prange L, Sośnica K, Mervart L, Jäggi A (2015) CODE's new solar radiation pressure model for GNSS orbit determination. J Geod 89(8):775791

Beutler G, Brockmann E, Gurtner W, Hugentobler U, Mervart L, Rothacher M, Verdun A (1994) Extended orbit modeling techniques at the CODE processing center of the international GPS service for geodynamics (IGS): theory and initial results. Manuscr Geod 19:367-386

Bock H, Dach R, Jäggi A, Beutler G (2009) High-rate GPS clock corrections from CODE: support of $1 \mathrm{~Hz}$ applications. J Geod 83(11):1083-1094

Dach R, Brockmann E, Schaer S, Beutler G, Meindl M, Prange L, Bock H, Jäggi A, Ostini L (2009) GNSS processing at CODE: status report. J Geod 83(3-4):353-365

Dach R, Lutz S, Walser P, Fridez P (2015) Bernese GNSS Software version 5.2. User manual, Astronomical Institute. University of Bern, Bern Open Publishing, Bern

Deng Z, Fritsche M, Uhlemann M, Wickert J, Schuh H (2016) Reprocessing of GFZ multi-GNSS product GBM. In: Proceedings of IGS workshop, Sydney, Australia

Dow JM, Neilan RE, Rizos C (2009) The international GNSS service in a changing landscape of global navigation satellite systems. J Geod 83(3-4):191-198

Duan B, Hugentobler U (2019) Precise orbit determination of Sentinel satellites using zero-difference ambiguity resolution approach. In: Living planet symposium, Milan, Italy

Duan B, Hugentobler U, Selmke I (2019) The adjusted optical properties for Galileo/BeiDou-2/QZS-1 satellites and initial results on BeiDou-3e and QZS-2 satellites. Adv Space Res 63(5):1803-1812

Ge M, Gendt G, Dick G, Zhang F (2005) Improving carrier-phase ambiguity resolution in global GPS network solutions. J Geod 79(1-3): 103-110

Ge M, Gendt G, Rothacher M, Shi C, Liu J (2008) Resolution of GPS carrier-phase ambiguities in precise point positioning (PPP) with daily observations. J Geod 82(7):389-399

Geng J, Bock Y (2013) Triple-frequency GPS precise point positioning with rapid ambiguity resolution. J Geod 87(5):449-460

Geng J, Shi C, Ge M, Dodson AH, Lou Y, Zhao Q, Liu J (2012) Improving the estimation of fractional-cycle biases for ambiguity resolution in precise point positioning. J Geod 86(8):579-589

Guo F, Zhang X, Wang J (2015) Timing group delay and differential code bias corrections for BeiDou positioning. J Geod 89(5):427445

Håkansson M, Jensen AB, Horemuz M, Hedling G (2017) Review of code and phase biases in multi-GNSS positioning. GPS Solut 21(3):849-860

Johnston G, Riddell A, Hausler G (2017) The international GNSS service. In: Teunissen PJ, Montenbruck O (eds) Springer handbook of global navigation satellite systems. Springer, Berlin, pp 967-982

Katsigianni G, Loyer S, Perosanz F (2019a) PPP and PPP-AR kinematic post-processed performance of GPS-only: Galileo-only and multiGNSS. Remote Sens 11(21):2477

Katsigianni G, Loyer S, Perosanz F, Mercier F, Zajdel R, Sośnica K (2019b) Improving Galileo orbit determination using zerodifference ambiguity fixing in a multi-GNSS processing. Adv Space Res 63(9):2952-2963 
Li P, Zhang X, Ren X, Zuo X, Pan Y (2016) Generating GPS satellite fractional cycle bias for ambiguity-fixed precise point positioning. GPS Solut 20(4):771-782

Li X, Li X, Yuan Y, Zhang K, Zhang X, Wickert J (2018) Multi-GNSS phase delay estimation and PPP ambiguity resolution: GPS, BDS, GLONASS, Galileo. J Geod 92(6):579-608

Li X, Li X, Liu G, Feng G, Yuan Y, Zhang K, Ren X (2019) Triplefrequency PPP ambiguity resolution with multi-constellation GNSS: BDS and Galileo. J Geod 93(8):1105-1122

Loyer S, Perosanz F, Mercier F, Capdeville H, Marty JC (2012) Zerodifference GPS ambiguity resolution at CNES-CLS IGS analysis center. J Geod 86(11):991-1003

Melbourne W (1985) The case for ranging in GPS based geodetic systems. In: Goad C (ed) Proceedings of the 1st international symposium on precise positioning with the global positioning system, NOAA, Rockville, Maryland, US Department of Commerce, pp 373-386

Montenbruck O, Hugentobler U, Dach R, Steigenberger P, Hauschild A (2012) Apparent clock variations of the Block IIF-1 (SVN62) GPS satellite. GPS Solut 16(3):303-313

Montenbruck O, Hauschild A, Steigenberger P (2014) Differential code bias estimation using multi-GNSS observations and global ionosphere maps. Navig J Inst Navig 61(3):191-201

Montenbruck O, Steigenberger P, Prange L, Deng Z, Zhao Q, Perosanz F, Romero I, Noll C, Stürze A, Weber G et al (2017) The multi-GNSS experiment (MGEX) of the international GNSS service (IGS)-achievements, prospects and challenges. Adv Space Res 59(7):1671-1697

Montenbruck O, Hackel S, Jäggi A (2018) Precise orbit determination of the sentinel-3a altimetry satellite using ambiguity-fixed GPS carrier phase observations. J Geod 92(7):711-726

Pan L, Zhang X, Li X, Liu J, Li X (2017) Characteristics of interfrequency clock bias for Block IIF satellites and its effect on triplefrequency GPS precise point positioning. GPS Solut 21(2):811822

Paziewski J, Wielgosz P (2015) Accounting for Galileo-GPS intersystem biases in precise satellite positioning. J Geod 89(1):81-93

Pearlman MR, Degnan JJ, Bosworth JM (2002) The international laser ranging service. Adv Space Res 30(2):135-143

Peter H, Jäggi A, Fernández J, Escobar D, Ayuga F, Arnold D, Wermuth M, Hackel S, Otten M, Simons W et al (2017) Sentinel-1A-first precise orbit determination results. Adv Space Res 60(5):879-892

Prange L, Orliac E, Dach R, Arnold D, Beutler G, Schaer S, Jäggi A (2017) CODE's five-system orbit and clock solution-the challenges of multi-GNSS data analysis. J Geod 91(4):345-360

Rodriguez-Solano CJ, Hugentobler U, Steigenberger P, Lutz S (2012) Impact of earth radiation pressure on GPS position estimates. J Geod 86(5):309-317

Schaer S, Villiger A, Arnold D, Dach R, Jäggi A, Prange L (2018) New ambiguity-fixed IGS clock analysis products at CODE. In: Proceedings of IGS workshop, Wuhan, China
Sleewaegen JM, Clemente F (2018) Quantifying the pilot-data bias on all current GNSS signals and satellites. In: Proceedings of IGS workshop, Wuhan, China

Steigenberger P, Montenbruck O (2017) Galileo status: orbits, clocks, and positioning. GPS Solut 21(2):319-331

Steigenberger P, Thoelert S, Montenbruck O (2018) GNSS satellite transmit power and its impact on orbit determination. J Geod 92(6):609-624

Teunissen P, Joosten P, Tiberius C (2003) A comparison of TCAR, CIR and LAMBDA GNSS ambiguity resolution. In: Proceedings of the 15th international technical meeting of the satellite division of the institute of navigation (ION GPS 2002) ION. Portland, Oregon

Thoelert S, Steigenberger P, Montenbruck O, Meurer M (2019) Signal analysis of the first GPS III satellite. GPS Solut 23(4):92

Uhlemann M, Gendt G, Ramatschi M, Deng Z (2015) GFZ global multiGNSS network and data processing results. In: IAG 150 years. Springer, pp 673-679

Villiger A, Schaer S, Dach R, Prange L, Sušnik A, Jäggi A (2019) Determination of GNSS pseudo-absolute code biases and their long-term combination. J Geod 93(9):1487-1500

Wang N, Yuan Y, Li Z, Montenbruck O, Tan B (2016) Determination of differential code biases with multi-GNSS observations. J Geod 90(3):209-228

Wubbena G (1985) Software developments for geodetic positioning with GPS using TI 4100 code and carrier measurements. In: Goad $\mathrm{C}$ (ed) Proceedings of the 1st international symposium on precise positioning with the global positioning system, NOAA, Rockville, Maryland, US Department of Commerce, pp 403-412

Xiao G, Li P, Sui L, Heck B, Schuh H (2019) Estimating and assessing Galileo satellite fractional cycle bias for PPP ambiguity resolution. GPS Solut 23(1):3 